\title{
PRIMARY SCHOOL PUPILS' MISCONCEPTIONS OF THE HUMAN RESPIRATORY SYSTEM IN PRIMARY SCHOOL STUDENTS: FROM IDENTIFICATION TO DECONSTRUCTION
}

\author{
Marina Silva ${ }^{1}$, António Almeida ${ }^{2}$ \\ ${ }^{1}$ Interdisciplinary Center of Educational Studies (PORTUGAL) \\ ${ }^{2}$ Polytechnic Institute of Lisbon / Interdisciplinary Center of Educational Studies / Research \\ Centre "Didactics and Technology in Education of Trainers" - University of Aveiro
}

(PORTUGAL)

\begin{abstract}
This study involved 38 students from the $6^{\text {th }}$ year of schooling attending a state school in Lisbon in which the Science syllabus is centered on the study of human body systems. Its purpose was to determine: (i) whether the students possessed any of the misconceptions related to the human respiratory system referenced in other studies (e.g. air enters and leaves our bodies with no function, air tubes connect the lungs to the heart, the air exhaled is rich in carbon dioxide and poor in oxygen); (ii) whether a learning design focused on the deconstruction of these misconceptions would be effective to the conceptual change of these pupils. To this end, the experimental group (class A) experienced a learning design expressly conceived for the deconstruction of the misconceptions related to the human respiratory system, while the control group (class B) experienced a traditional approach with interactive lectures and use of the school textbook. The data were collected through a questionnaire administered in two stages (pre and post-test) and a t-test was used to compare the means obtained by the two groups in the pre-test and to verify the gains of each class between the pre and the post-test. After analysis of the results of the pre-test, it was found that the students of the two groups had several misconceptions of the human respiratory system. The experimental group had more significant gains, although the students in both groups reduced their misconceptions. Both results support the idea that formal education, if provided with well-structured activities, can contribute to the conceptual restructuring of the students' mind, albeit with a different rate of success. Finally, the present study also helps to confirm the universality of certain misconceptions.
\end{abstract}

Keywords: Primary School, science education, human respiratory system, misconceptions, strategies for conceptual change.

\section{INTRODUCTION}

Scientific learning has been considered as one of the essential dimensions in the training of young people, since today's society is characterized by unprecedented scientific and technological progress. However, this learning is affected by incorrect ideas (misconceptions) that students already have, which often correspond to superficial and intuitive explanations of the phenomena that surround them.

Therefore, misconceptions are ideas that appear as an alternative to accepted scientific concepts, which can not be seen as distractions, lapses of memory or miscalculations, but as potential explanatory models resulting from a conscious effort to theorize natural phenomena ([1] Martins et al. 2006). Learners organize their own daily experiences and interpret the reality around them. These are based on many notions of common sense that clearly influence the new school learning ([2] Duit, 1991; [3] Afonso \& Neves, 2000; [4] Ramos, 2009).

[5] Furió, Solbes \& Carrascosa (2006) systematize some of the features of these misconceptions: (i) they present an internal logic and provide explanations that make sense to learners; so, school knowledge does not build from zero, since students already possess previous knowledge; (ii) they persist in time and are difficult to deconstruct merely via traditional teaching, through transmission from teacher to leaner; (iii) they interact with the teaching-learning process and hamper the correct understanding of science concepts.

Misconceptions may have different origins. [5] Furió, Solbes \& Carrascosa, (2006) highlight the following: sensorial - relations of cause and effect based on the observed reality; cultural - associated with the phenomena that children know and with the reality that surrounds them; scholar - associated with simplifications of complex concepts present in school textbooks or addressed by teachers. The 
influence of school context in the building of misconceptions has been pointed out by several authors (e. g., [6] Matthews \& Davies, 1999; [7] Köse, 2008; [8] Kurt, et al., 2013). The use of didactic models not properly interpreted by the students can induce misconceptions. In fact, didactic models are simplifications of the reality and can help the comprehension of complex phenomena that, otherwise, could be difficult for students to understand. However, since models use and promote analogical thinking, it is strong the possibility of helping the development of wrong ideas, especially when teachers are not conscious of this possibility. Still in the field of school context, the non-identification of the previous ideas of the students by the teachers can help to accentuate many misconceptions.

But [6] Matthews and Davies (1999) also highlight the role of media in the construction of misconceptions due to the information they transmit. In fact, films, cartoons, and even the news can be sources of misconceptions due to wrong ideas that are broadcast. But even when the information is correct it can also be misunderstood by the pupils. Whatever the path that leads to student misconceptions, teachers must depart from students' knowledge to address new knowledge and establish new conceptual connections ([9] Michael, 1998; [8] Kurt et al., 2013).

For the identification of pupils' misconceptions, teachers must give opportunities in class for them to present their own ideas. Hence, teachers with a constructivist perspective of the teaching / learning process can more easily identify the misconceptions of their students than teachers with empiricist beliefs, because the former will find it easier to devise strategies that require a greater exposure of the students and don't possess the naive idea that facts can be absorbed in a simple way.

Misconceptions can be identified using questionnaires, interviews, analysis of drawings, concept maps, practical activities or simply during dialogues in the classroom. It is then up to the teacher to outline the most appropriate didactic strategies. To do this, it helps to understand the roots of the misconceptions of their students and to not be surprised by their resurgence, since the process of deconstruction is not always easy and permanent.

For significant learning to take place, the teacher should try to contribute to changing the conceptual structures of the students, reorganizing them and promoting their modification. As stated by [10] Cachapuz, et al., 2002), it is the teacher's role to be an organizer of intentional strategies that provoke cognitive conflict; this conflict provokes doubts in the students and challenges their way of thinking.

Based on the ideas expressed above, the present study had the following objectives:

1 To identify misconceptions of the human respiratory system, determining whether they were similar to those previously mentioned in other studies with children of the same age;

2 To teach the human respiratory system using a systematic confrontation between these misconceptions and the correct scientific concepts;

3 To promote different activities which induce cognitive conflict in pupils;

4 To check conceptual changes three weeks after completing the learning design process.

The misconceptions dealt with in class were the following:

1 Air only enters and leaves our bodies, serving no physiological function ([11] Allen, 2014); we breathe only to survive ([12] Bajd, Praprotnik \& Matyàsek, 2010; [13] Garcia-Barros, MartínezLosada \& Garrido, 2011; [8] Kurt et al., 2013);

2 Air tubes connect the lungs to the heart ([11] Allen, 2014);

3 The air exhaled is rich in carbon dioxide and poor in oxygen; we inhale oxygen and exhale carbon dioxide ([14] Boo, 2005; [12] Bajd, Praprotnik \& Matyàsek, 2010).

\section{METHODOLOGY}

The present study used a quasi-experimental design, with an experimental group and a control group. The methodology had a predominantly quantitative content. The two classes were made up of 19 students each, and with the following similar features: medium to low socioeconomic level; medium to low school achievement; several children had repeated at least one school year; presence of students with special educational needs; mean age of 12 years; a similar ratio of boys to girls.

The students were in the $6^{\text {th }}$ year of schooling in a state school in Lisbon. The Science syllabus of this year of schooling is centered on the study of human body systems. However, this subject is also present in previous years of schooling, although approached at a more basic level. Although the 
choice of school was not totally random in the universe of primary schools in the city, the choice was random in the set of ten basic schools that collaborate with an institution of higher education in the initial training of teachers.

A questionnaire was applied to both groups in two stages (pre and post-test). The questionnaire consisted of open and closed questions divided into three parts, and its content was related to misconceptions of the respiratory system revealed in other studies in different cultural contexts. The questionnaire was validated by two experts in Didactics of Sciences and was tested in a pilot sample of 15 pupils from another class with similar features. From this process several changes were introduced, especially those related to the simplification of the terms included. Firstly, the terms of inspiration and expiration in the pulmonary ventilation present in the initial version of the questionnaire were unknown by the pupils and were substituted by more common ways of describing the same processes: "the air that we breathe in" and "the air that we breathe out". Secondly, the exclusion of the chemical formulas to designate the gases present in the human respiratory process was also done. And finally, we decided to read aloud each question during the administration of the questionnaire to facilitate its understanding. The final structure of the questionnaire is shown in Table 1.

Table 1. The structure of the questionnaire.

Part I

1-Which of the following organs form part of the human respiratory system?

Heart, nose, esophagus, lungs, trachea, stomach, larynx, intestine, mouth, pharynx, anus, bronchioles, liver, diaphragm.

2- On the scheme of the human body: a) draw the organs that are part of the respiratory system; b) mark the way that air travels when it enters the organism.

Part II

Rate the following statements as true or false, always justifying your choices.

1-We should breathe through the mouth, since it admits more air in the organism.

2-Air is just breathed in and out, serving no function in the organism.

3-The lungs are connected to the heart by tubes where the air circulates.

4-The gases that we breathe in are the same as the gases that we breathe out.

5 -From the gases that we breathe in, oxygen is the most important for the respiratory function.

6 -Gas exchanges occur in the heart.

7-Carbon dioxide is the only gas that we breathe out.

8-Oxygen is the only gas that we breathe in.

9-The heart is one of the organs of the respiratory system

10-Of the air that we breathe in, carbon dioxide is the most important for the respiratory function

Part III

Circle the correct word in order to make true statements

1-The air that we breathe in has more / less oxygen than the air that we breathe out.

2-The air that we breathe in has more / less carbon dioxide than the air that we breathe out.

3-The air that we breathe out has more / less oxygen than carbon dioxide.

4-The air that we breathe in has more / less oxygen than carbon dioxide.

The total score for the questionnaire was $100 \%$, as if it were a test, with a mark awarded for each question. All the incorrect ideas expressed by the pupils were collected to be used during the intervention stage as discussion topics. In the questions where students were asked to evaluate the veracity of statements, a justification was always required. The question was only considered correct when the justification was consistent with the assessment. 
The experimental group was set, between the pre and the post-test, a sequence of activities expressly designed for the deconstruction of the misconceptions revealed (Table 2); the control group had a more traditional approach to the respiratory system with interactive lectures and the use of the school textbook.

Table 2. Activities set to the experimental group.

The intervention design set to the experimental group

1. A PowerPoint presentation with a selection of the more frequent misconceptions revealed by the students. Discussion in a large group about the ideas put forward.

2. A PowerPoint presentation with a problem situation regarding the saving of lives through the use of mouth-to-mouth resuscitation, in order to deconstruct the idea that we only breathe out carbon dioxide.

3. A Prezi presentation with figures, tables, and images to discuss more misconceptions now related mainly to the composition of the air that we breathe in and out.

4. A practical activity of observation of the organs of the respiratory system and the heart of a pig, to deconstruct the misconception that the heart is an organ of the respiratory system.

Pre and post-test results in both groups were compared using inferential statistics. The skewness of the results was first evaluated and found to be in a normal distribution. Accordingly, a t-test was used to compare the means obtained by the two groups in the pre-test and to verify the gains of each class between the pre and the post-test. The differences between the groups at the initial moment were statistically non-significant $(p=0.410)$, an important aspect to better appreciate the gains of each group between the two stages of the administration of the questionnaire.

\section{RESULTS}

After analysis of the results of the pre-test, it was found that, as previously mentioned, the students of the two groups had several misconceptions of the human respiratory system. Table 4 summarizes some of the results obtained by both groups (experimental and control groups) in the pre-test and in the post-test and only considers the items of the questionnaire most directly related to the misconceptions that were intended to be deconstructed.

The most relevant changes are the following: a decrease in the misconception that the heart is part of the respiratory system, more pronounced in the experimental group; a decrease in both groups of the idea that the gases that we breathe in are not the same as the gases that we breathe out; a pronounced decrease only in the experimental control of the idea that the air that we breathe out has less oxygen than carbon dioxide. Even so, a few misconceptions continue in the pupils of both groups with a high percentage, even considering some improvements at least in one of the groups. That is the case of the idea that lungs are connected to the heart by air tubes, or the idea that the gases that we breathe in are not the same gases that we breathe out.

Globally, from the analysis of the results present in Table 4, it is possible to conclude that there is a decrease in the percentage of students that revealed misconceptions in both groups, but the decrease is more accentuated in the experimental group. In a few misconceptions, the control group got poorer results.

In general, there was also a great evolution in the drawings made by the students of both classes, in which they had to indicate and label the organs of the human respiratory system, as well as the way the air travels through the organism, a task set in the first part of the questionnaire. 
Table 4. Percentage of the pupils from the Experimental Group (EG) and Control Group (GC) revealing misconceptions in the pre-test (pre-t) and in the post-test (pos-t) at the two stages (1 and 2) of eight of the items of the questionnaire more related with the misconceptions to be deconstructed.

\begin{tabular}{l|c|c|c|c}
\hline \hline & \multicolumn{2}{|c|}{$\mathrm{GE} \mathrm{n}=19$} & \multicolumn{2}{|c}{$\mathrm{GC} \mathrm{n}=19$} \\
\hline Items of the questionnaire & Pre-t1 & Pos-t2 & Pre-t1 & Pos-t2 \\
\hline \begin{tabular}{l} 
1. The heart is part of the respiratory system. \\
\hline 2. The lungs are connected to the heart by air tubes.
\end{tabular} & 52.63 & 15.79 & 73.68 & 63.16 \\
\hline $\begin{array}{l}\text { 3. Air is just breathed in and out, serving no function in the organism. } \\
\text { 4. The gases that we breathe in are not the same gases as we }\end{array}$ & 36.84 & 21.05 & 21.05 & 5.26 \\
\hline $\begin{array}{l}\text { breathe out. } \\
\text { 5. The air that we breathe in has less oxygen that the air that we } \\
\text { breathe out. }\end{array}$ & 21.06 & 5.26 & 26.32 & 15.79 \\
\hline $\begin{array}{l}\text { 6. The air that we breathe in has more carbon dioxide than the air we } \\
\text { breathe out. }\end{array}$ & 21.06 & 15.79 & 47.37 & 26.32 \\
\hline $\begin{array}{l}\text { 7. The air that we breathe out has less oxygen than carbon dioxide. } \\
\text { 8. The air that we breathe in has less oxygen than carbon dioxide. }\end{array}$ & 68.43 & 26.32 & 52.63 & 68.42 \\
\hline \hline
\end{tabular}

From the comparative analysis of the means obtained in the pre and the post-test by the experimental group and the control group, both groups had statistically significant differences (respectively $p=$ 0.000 and $p=0.004$ ). Thus, students from both classes decreased the incidence of misconceptions, but the improvement of the experimental group was more significant. It was found that the mean of gains of the experimental class was 25.37 and that of the control group 8.89. From the application of a t-test again, there is a significant difference between the gains of the two groups $(p=0.000)$.

\section{CONCLUSIONS}

The schooling process includes learning a multiplicity of science concepts from different sciences, mainly from physics, chemistry, biology, geology. For many of these concepts, students have their own ideas distinct from the scientific explanation, the so-called misconceptions, which interfere negatively with learning.

This study allows us to favor the efficacy of a design learning process paying particular attention to the identification and deconstruction of several misconceptions specifically related to the human respiratory system. Even so, the process experienced by the control group brought some positive results and seems also to have facilitated a decrease in the incidence of certain misconceptions. Both results support the idea that formal education, if provided with well-structured activities, can contribute to the conceptual restructuring of the students' mind, albeit with a different rate of success.

Therefore, the research carried out also highlighted the importance of a learning process more centered on the learners, promoting the confrontation of ideas in the classroom and exposing pupils to the incoherence of certain of their conceptions. It highlighted the role of practical activities in the deconstruction of misconceptions, also helping pupils to discover how their previous ideas can lose their explanatory sense. Thus, a greater exposure of students' ideas in the course of their learning process, and presenting them with situations that can promote cognitive conflict, are more likely to help pupils to overcome a variety of misconceptions. Of course, the use of all these strategies, which help the deconstruction of misconception, implies time, a dimension that is always missing when the process of teaching and learning is conditioned by the existence of exams.

Another important aspect of the results is the following: it remains to be seen whether the persistence of the change will occur equally in both groups, knowing that misconceptions often reappear after a cessation of contact with the formal teaching of the issues learned in school ([15] Pozo \& Gómez Crespo, 2009). Therefore, in future studies it will be relevant to implement a research design that also includes a new administration of the questionnaire several months after the end of the formal approach to the scientific issue considered. 
Finally, the present study attempts to confirm, as [11] Allen (2014) points out, the universality of certain misconceptions. If some of them may be heavily influenced by the cultural context of the students, others seem to be found in samples from different countries, proving that some ideas are common in the human mind independently of the particularities of each context. For all these reasons, the present study can help the future approach of the issue in discussion, helping teachers from different places and cultures to design the best strategies to promote the changing of wrong ideas revealed by students, contributing to a better understanding of different scientific concepts.

\section{ACKNOWLEDGEMENTS}

This study is part of a project entitled "Research in didactics of science: implications for the improvement of teaching practices" and it is funded by the Interdisciplinary Center of Educational Studies (Centro Interdisciplinar de Estudos Educacionais - CIED) seated in Lisbon School of Education, Portugal.

\section{REFERENCES}

[1] I. P. Martins, M. L. Veiga, F. Teixeira, C. Tenreiro-Vieira, R. M. Vieira, A. V. Rodrigues, F. Couceiro, Educação em Ciências e Ensino Experimental: Formação de professores. Lisboa: Ministério da Educação, 2006.

[2] R. Duit, "On the role of analogies and metaphors in learning science," Science Education, vol. 75, no. 6, pp. 649-672, 1991.

[3] M. Afonso \& I. Neves, "Influência da prática pedagógica na mudança conceptual em ciências: Um estudo sociológico," Revista Portuguesa de Educação, vol. 13, no. 1, pp. 247-282, 2000.

[4] M. S. Ramos, Teoria do Caos: Potencialidades na modelização da aprendizagem de conceitos científicos, Lisboa: Edições Colibri, 2009.

[5] C. Furió, J. Solbes \& J. Carrascosa, "Las ideas alternativas sobre conceptos científicos: Tres décadas de investigación," Alambique: Didáctica de las ciencias experimentales, vol. 48, pp. 64-77, 2006.

[6] B. Matthews \& D. Davies, "Changing children's images of scientists: Can teachers make a difference?" School Science Review, vol. 80, no. 293, pp. 79-85, 1999.

[7] S. Köse, "Diagnosing student misconceptions: Using drawings as a research method," World Applied Sciences Journal, vol. 3, no. 2, pp. 283-293, 2008.

[8] H. Kurt, G. Ekici, M. Aktas \& Ö. Aksu, "On the concept of "Respiration": Biology student teachers' cognitive structures and alternative conceptions," Educational Research and Reviews, vol. 8, no. 21, pp. 2101-2121, 2013.

[9] J. A. Michael, "Students' misconceptions about perceived physiological responses," Advances in Physiology Education, vol. 19, no. 1, pp. 90-98, 1998.

[10] A. Cachapuz, J. Praia \& M. Jorge, Ciência, Educação em Ciência e Ensino das Ciências. Lisboa: Ministério da Educação, 2002.

[11] M. Allen, Misconceptions in Primary Science. Maidenhead (UK): Open University Press, 2014.

[12] B. Bajd, L. Praprotnik, \& J. Matyàsek, "Students' Ideas about Respiration: A Comparison of Slovene and Czech Students," School and Health, vol. 21, pp. 245-251, 2010.

[13] S. García-Barros, C. Martínez-Losada \& M. Garrido, "What do children aged four to seven know about the digestive system and the respiratory system of the human being and of other animals?", International Journal of Science Education, vol. 33, no.15, pp. 2095-2122, 2011.

[14] H. K. Boo, "Teachers' misconceptions of biological science concepts as revealed in science examination papers," Australian Association for Research in Education Conference, Parramatta: Australia, 2005.

[15] J. I. Pozo \& M. A. Gómez Crespo, Aprender y enseñar ciencia. Madrid: Ediciones Morata, 2009. 